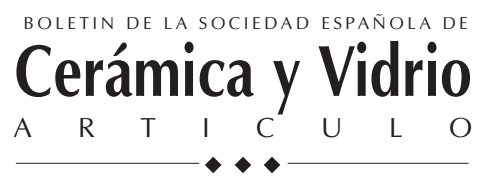

\title{
Óxidos de estaño: nuevos anodos para baterías de Ion-Litio
}

\author{
J. MORALES Y L. SÁNCHEZ* \\ Laboratorio de Química Inorgánica, Facultad de Ciencias, Universidad de Córdoba. \\ Avda. S. Alberto Magno, s/n. 14004, Córdoba. Spain.
}

\begin{abstract}
La reacción entre el litio y óxidos ó aleaciones de estaño es reversible cuando se realiza por métodos electroquímicos y a bajos potenciales con respecto al electrodo de litio. Esta propiedad permite el uso de estos materiales como electrodos negativos en el diseño y construcción de baterías tipo ion-litio. En el caso de óxidos de estaño la reacción de inserción de litio en la matriz conduce a la formación de aleaciones Li-Sn. La presencia de agregados grandes de átomos de estaño, formados durante la inserción de litio, origina la fragmentación y el desmoronamiento del material anódico. Este efecto provoca la pérdida del contacto eléctrico entre partículas, con el consiguiente deterioro de la batería. La optimización del rendimiento electroquímico de estos materiales se puede lograr mediante el control del crecimiento y tamaño de los agregados de estaño.
\end{abstract}

Palabras clave: electroquímica, litio, óxidos de estaño, batería ion-litio.

Tin Oxides: New anodes for Li-Ion cells.

The electrochemical reaction between $\mathrm{Li}$ and tin-based compounds, is reversible and takes place at low potentials measured against the Li electrode. This feature allows the use of these materials as negative electrodes in the manufacturing of Li-ion batteries. The lithium insertion in the tin oxides leads to the formation of Li-Sn alloys. The presence of large tin clusters, formed during the reaction, causes the cracking and crumblimg of the anodic material and the electronic contact between the particles is lost. The electrochemical performance of these materials can be optimized by controlling the growing and size of tin aggregates.

Keywords: electrochemistry, lithium, tin oxides, Li-ion battery.

\section{INTRODUCCIÓN}

Las baterías de ion-litio constituyen hoy en día la principal fuente de energía eléctrica necesaria para el funcionamiento de diferentes equipos electrónicos portátiles como teléfonos, ordenadores, cámaras de vídeo, etc., gracias a los altos valores de densidad de energía específica y volumétrica que son capaces de suministrar. Estas baterías fueron comercializadas a partir de 1990, por la firma Sony Energetics Inc., usando en su diseño carbón como electrodo negativo. La elección de este material fue consecuencia de los numerosos y exhaustivos estudios realizados en diversos sistemas basados en el elemento carbono, como el grafito, coke y carbones de baja cristalinidad, actuando como ánodos en baterías recargables de litio (1). Estos materiales presentan la capacidad de almacenar iones litio de forma reversible entre las láminas de átomos de carbono y desarrollar un potencial lo suficientemente bajo, en relación al par $\mathrm{Li} / \mathrm{Li}^{+}$. Ambas características les permite actuar como el electrodo negativo en baterías ion-litio. La capacidad teórica máxima que proporciona el grafito se calcula a partir de la inserción de un litio por cada seis átomos de carbono $\left(\mathrm{LiC}_{6}, 372 \mathrm{Ah} / \mathrm{kg}\right)$.

$\mathrm{Si}$ bien estos materiales carbonáceos cumplen los requerimientos necesarios para su uso en pilas comerciales, presentan ciertas limitaciones, entre las que cabe destacar i) pérdida de material debido a la intercalación de litio con el consiguiente hinchamiento y aumento significativo del parámetro de red; ii) deterioro del electrodo como consecuencia de fenómenos de cointercalación del disolvente, por ejemplo carbonato de propileno; y iii) en aquellos materiales carbonáceos menos sensibles a sufrir procesos de cointercalación, pérdida de capacidad después de la primera intercalación de litio. Estas limitaciones han motivado un interés científico creciente en el desarrollo de materiales de inserción alternativos que puedan actuar como electrodos negativos. Los primeros estudios se centraron en óxidos metálicos con bajos potenciales redox frente al litio, por ejemplo, $\mathrm{MoO}_{2}, \mathrm{WO}_{2}, \mathrm{TiO}_{2}, \mathrm{Fe}_{2} \mathrm{O}_{3}$, si bien, estos materiales no representan una clara competencia para el carbón (2). En 1994, investigadores de Fuji Photo Film Co. Ltd., anunciaron el desarrollo de una nueva clase de baterías tipo ion-litio en las que se utilizan óxidos de estaño como materiales electródicos negativos, en sustitución del carbón o grafito (3). Estos nuevos ánodos están constituidos por compuestos amorfos inorgánicos que contienen elementos pertenecientes a los grupos 13, 14 y 15. Los resultados más destacables han sido los obtenidos en óxidos de estaño $\left(\mathrm{SnO}, \mathrm{SnO}_{2}\right)$ y óxidos mixtos amorfos de estaño (TCO: Tin Oxide Composites). Aún cuando en trabajos anteriores se había demostrado que el $\mathrm{SnO}_{2}$ podía insertar 
pequeñas cantidades de litio $(4,5)$, los resultados obtenidos por Idota y col. (3) presentan como gran novedad la capacidad que poseen estos ánodos de incorporar hasta 6 átomos de litio por átomo de estaño, que corresponde a una capacidad específica teórica de unos $1030 \mathrm{Ah} / \mathrm{kg}$., unas tres veces superior a la que exhibe el carbón. Aunque la capacidad inicial decrece en torno a un $37 \%$ en los primeros ciclos, la capacidad reversible de almacenaje de litio se estabiliza en valores próximos a 600 $\mathrm{Ah} / \mathrm{kg}$, valor que puede mantenerse durante la actuación de la batería. Como consecuencia de estas propiedades sobresalientes se ha suscitado un gran interés en el estudio de los óxidos de estaño como materiales anódicos en baterías de litio.

La presente comunicación se ha planteado a modo de una revisión del estado actual de este interesante campo de investigación, incluyendo los resultados recientemente obtenidos por nuestro grupo de trabajo. Se pretende que el lector adquiera una visión clara y actualizada sobre la problemática y el empleo de materiales basados en óxidos de estaño como electrodos negativos en baterías de ion-litio.

\section{COMPORTAMIENTO ELECTROQUIMICO DE OXIDOS DE ESTAÑO EN CELDAS DE LITIO.}

\subsection{Mecanismo de inserción de litio en óxidos de estaño.}

Se posee un conocimiento bastante preciso de la reacción de inserción electroquímica de litio en matrices de $\mathrm{SnO}$ y $\mathrm{SnO}_{2}$ obtenido de estudios termodinámicos $(6,7)$, así como de la aplicación de una amplia variedad de técnicas físicas para identificar los intermedios formados (8-11). Tres reacciones principales tienen lugar cuando se parte de $\mathrm{SnO}_{2}$ como matriz de inserción (8):

$$
\begin{aligned}
& \mathrm{SnO}_{2}+2 \mathrm{Li}^{+}+2 \mathrm{e}^{-} \rightarrow \mathrm{SnO}+\mathrm{Li}_{2} \mathrm{O} \\
& \mathrm{SnO}^{-} 2 \mathrm{Li}^{+}+2 \mathrm{e}^{-} \rightarrow \mathrm{Sn}+\mathrm{Li}_{2} \mathrm{O} \\
& \mathrm{Sn}^{\circ}+\mathrm{Li}^{+}+\mathrm{xe}^{-} \rightarrow \mathrm{Li}_{\mathrm{x}} \mathrm{Sn}
\end{aligned}
$$

En la Figura 1 se recogen los potenciales de equilibrio de todas las reacciones posibles junto con la curva galvanostática de descarga del $\mathrm{SnO}_{2}$. Las dos primeras reacciones tienen lugar a 1.88 y $1.58 \mathrm{~V}$, frente al electrodo de litio, respectivamente, y el proceso de reducción $\mathrm{Sn}^{4+} \rightarrow \mathrm{Sn}^{2+}$ sólo se ha podi-

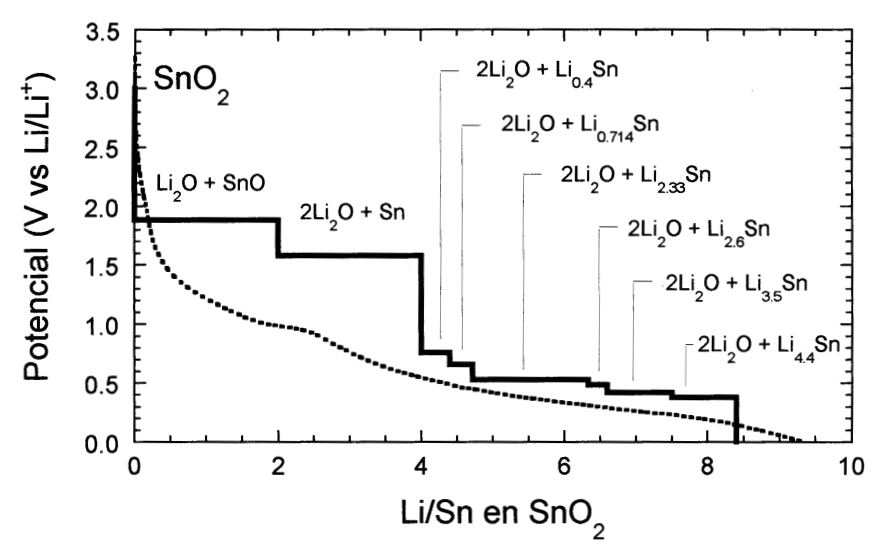

Figura 1: Curva teórica de potencial para el sistema $\mathrm{Li} / \mathrm{SnO}_{2}$. La línea discontínua representa la curva de potencial correspondiente a la primera descarga realizada en una celda $\mathrm{SnO}_{2} / \mathrm{LiPF}_{6}$ (EC-DMC)/Li (23). do detectar para bajos contenidos de litio (4). Cuando la cantidad de iones $\mathrm{Li}^{+}$transferida es grande, el estaño metálico formado ha sido detectado por DRX (8) y espectroscopia Raman (9). $\mathrm{El} \mathrm{Li}_{2} \mathrm{O}$ es amorfo, según revelan los estudios de $\mathrm{RMN}$ de ${ }^{7} \mathrm{Li}(10,11)$. Estas dos reacciones son irreversibles, de tal modo que el oxígeno que forma el $\mathrm{Li}_{2} \mathrm{O}$ no se puede volver a utilizar para regenerar los óxidos de estaño. Esto implica que cuatro átomos de litio no son recuperables en el proceso de carga, dos si partimos de una matriz de $\mathrm{SnO}$, lo que representa una pérdida de capacidad de unos $711 \mathrm{Ah} / \mathrm{kg}$. Esta capacidad irreversible adquiere una importancia significativa y se ha de tener en cuenta a la hora de fabricar una batería de ion-litio. Para compensar la pérdida de capacidad es necesario disponer de una cantidad extra de electrodo positivo, que es el que suministra los iones $\mathrm{Li}^{+}$al material anódico, con el consiguiente aumento de tamaño y peso del electrodo, y de la propia batería.

El interés de estos materiales electródicos radica principalmente en la alta capacidad reversible que presentan y que es consecuencia de la reacción [3]. De este modo, cuando todo el oxígeno presente ha sido consumido en la formación de $\mathrm{Li}_{2} \mathrm{O}$, el estaño metálico originado puede reaccionar hasta con un máximo de 4.4 átomos de litio, que son empleados en formar sucesivas aleaciones de composición $\mathrm{Li}_{0.4} \mathrm{Sn}, \mathrm{Li}_{0.714} \mathrm{Sn}, \mathrm{Li}_{2.33} \mathrm{Sn}, \mathrm{Li}_{2.6} \mathrm{Sn}, \mathrm{Li}_{3.5} \mathrm{Sn}$ y $\mathrm{Li}_{4.4} \mathrm{Sn}$, algunas detectadas por estudios in situ de DRX (8). Estos compuestos laminares de litio son fases estructuralmente relacionadas, lo que permite que la inserción de litio sea un proceso reversible. En diferentes publicaciones $(12,13)$ se ha destacado la importancia de la presencia de $\mathrm{Li}_{2} \mathrm{O}$ durante la etapa de formación de las diferentes aleaciones Li-Sn. En este proceso, las fases Li-Sn sufren grandes cambios de volumen, pudiendo ocurrir la agregación de estaño metálico en grandes dominios coherentes conforme aumenta el contenido de litio (Figura 2). Así, el $\mathrm{Li}_{2} \mathrm{O}$ ejerce una doble función: i) actúa como "adhesivo" para mantener unidas las partículas de Li-Sn asegurándose el contacto eléctrico entre ellas, y a su vez ii) ayuda a la reversibilidad de las aleaciones, actuando en contra de la fragmentación y desmoronamiento originados por los cambios de volumen.

\subsection{Optimización de los óxidos de estaño como materiales electródicos negativos.}

\subsection{1 ÓXIDOS DE ESTAÑO.}

El uso de óxidos de estaño como ánodos en baterías de litio requiere una optimización de las condiciones de trabajo que



Figura 2: Idealización del proceso de inserción de litio en $\mathrm{SnO}_{2}$. 
permita un mejor aprovechamiento de la capacidad específica reversible que presentan estos electrodos, originada por la inserción/ desinserción de litio en su estructura. Con este objetivo, Courtney y col. (12) han sido los primeros investigadores que han realizado un seguimiento riguroso de la ciclabilidad que presenta este material en función de los distintos intervalos de potencial en los que se pueden formar las aleaciones LiSn. La selección de ventanas de potencial adecuadas, en las cuales se realiza el proceso electroquímico de inserción/ desinserción de litio, permite disminuir el tamaño de los agregados de átomos de estaño. Cuando el proceso de reducción se realiza hasta potencial cero, se produce la aleación $\mathrm{Li}_{4.4} \mathrm{Sn}$, composición que corresponde a la máxima capacidad de inserción de estos materiales, y por ello es utilizado como límite inferior de la ventana de potencial. En cambio, el límite del potencial de oxidación parece desempeñar un papel más relevante. Los procesos de oxidación que sobrepasan un potencial de $0.8 \mathrm{~V}$ implican la formación de estaño metálico, y una oxidación superior a $1.3 \mathrm{~V}$ podría provocar la destrucción de la matriz de $\mathrm{Li}_{2} \mathrm{O}$ que separa las regiones de estaño metálico. El estaño metálico es dúctil y posee un punto de fusión bajo, lo que sugiere una buena movilidad atómica a temperatura ambiente, mientras que las fases de Li-Sn poseen mayores puntos de fusión que el estaño (14) y son más quebradizas. Por consiguiente, una oxidación electroquímica hasta el límite de voltaje en el que se forma estaño metálico favorece claramente el proceso de agregación de estaño. Un aumento excesivo en el tamaño de estos agregados origina la coexistencia de aleaciones que poseen estructuras cristalinas y relaciones Li/Sn diferentes dentro de la misma partícula, observándose regiones multifases cuyas diferencias de volumen molar producen una caída de la capacidad. Además, las regiones dentro de una partícula pueden quedar aisladas eléctrica e iónicamente y se vuelven inactivas. Por esta razón, cuando estos compuestos actúan frente al electrodo de litio el rango de potencial de trabajo más adecuado es $1.0-0 \mathrm{~V}$.

Los argumentos expuestos justifican que el control del crecimiento de los agregados de estaño es un aspecto importante para obtener una buena ciclabilidad en estos compuestos, y en esta dirección se han centrado los esfuerzos científicos dirigidos a la optimización de óxidos de estaño como materiales anódicos.

Idota y col. $(3,10)$ fueron los primeros en diseñar óxidos compuestos de $\mathrm{Sn}, \mathrm{B}, \mathrm{P}$ y $\mathrm{Al}$, amorfos y de composición $\mathrm{Sn}_{1.0} \mathrm{~B}_{0.56} \mathrm{P}_{0.40} \mathrm{Al}_{0.42} \mathrm{O}_{0.36}$. Cuando este ánodo trabaja en una ventana de potencial comprendida entre 1.2 - $0 \mathrm{~V}$ es capaz de desarrollar una capacidad de almacenamiento de ion litio de unos $600 \mathrm{Ah} / \mathrm{kg}$. Óxidos similares, de estequiometría SnO: $\left(\mathrm{B}_{2} \mathrm{O}_{3}\right)_{\mathrm{x}}:\left(\mathrm{P}_{2} \mathrm{O}_{5}\right)_{\mathrm{y}}$ (con valores de $\mathrm{x}$ e y comprendidos entre 0.1 y 0.4$)$, fueron estudiados por Courtney y col. $(12,13)$ mostrando una capacidad reversible de unos $500 \mathrm{Ah} / \mathrm{kg}$, valor que se mantiene constante para los primeros ciclos. La reacción electroquímica de inserción de litio en este electrodo se puede expresar de la siguiente manera (12):

$$
4 \mathrm{Li}^{+}+\mathrm{Sn}_{2} \mathrm{BPO}_{6}+4 \mathrm{e}^{-} \rightarrow 2 \mathrm{Li}_{2} \mathrm{O}+2 \mathrm{Sn}^{\circ}+1 / 2 \mathrm{~B}_{2} \mathrm{O}_{3}+1 / 2 \mathrm{P}_{2} \mathrm{O}_{5}
$$

Esta reacción nos muestra como, en comparación con el $\mathrm{SnO}_{2}$, disminuye la relación $\mathrm{Li} / \mathrm{Sn}$ necesaria para la formación del óxido de litio, lo que significa que estos materiales presentarán una menor capacidad inicial irreversible. Además, si consideramos que los óxidos de boro y fósforo no reaccionan con el litio, al igual que el $\mathrm{Li}_{2} \mathrm{O}$, dispersarían los átomos de estaño, dificultando el movimiento de estos átomos para la formación de los agregados. Una alta relación material dispersante/ estaño provoca que los átomos de estaño deban recorrer grandes distancias para poder agregarse en clusters de igual tamaño. Este comportamiento se refleja en un mejor mantenimiento de la capacidad reversible con el número de ciclos, aún cuando, se sacrifica la capacidad específica del material puesto que existe un aumento de su masa molecular al añadir una elevada cantidad de material inactivo (13). Por otra parte, los materiales amorfos representarían el límite de tamaño de grano más pequeño, en el supuesto de que durante el ciclaje de la batería los átomos de estaño no se agregaran. De hecho, como se ha demostrado en ánodos de $\mathrm{SnO}_{2}$, la reversibilidad de la reacción con litio aumenta cuando disminuye el tamaño de los cristales (12).

En estos materiales compuestos de Sn-B-P-O, también se observa como los clusters de estaño aumentan de tamaño al incrementar el número de ciclos electroquímicos a que es sometido el material anódico. Al cabo de un cierto número de ciclos se alcanza un tamaño definitivo cuyo volumen está relacionado con la fracción de estaño que exista en el compuesto y con la distancia cluster-cluster, la cual ha de ser siempre más pequeña que la distancia máxima que puedan recorrer los átomos de estaño en su movimiento entre clusters (13).

Hasta ahora hemos descrito el buen comportamiento electroquímico de los ánodos TCO. No obstante, estudios de reciente aparición cuestionan en cierto modo su aplicabilidad como electrodos negativos en baterías recargables de litio, porque no alcanzan a mantener valores constantes de capacidad reversible cuando son extensamente ciclados. En este sentido, Machill y col. (15) han investigado las propiedades electroquímicas del sistema Sn-B-P-O, y si bien obtienen muestras originales de diferente cristalinidad a las de las referencias 10,12 y 13, los resultados obtenidos se interpretan admitiendo que parte del estaño aparece como $\mathrm{SnP}_{2} \mathrm{O}_{7}$, material que es inactivo para el almacenamiento de litio y cuya formación debe evitarse.

La microscopía electrónica de transmisión de alta resolución (HRTEM) también ha aportado nuevos datos que explicarían la pérdida continua de capacidad reversible en los ánodos de estaño. A través de imágenes obtenidas sobre muestras de $\mathrm{SnO}(16)$ y $\mathrm{SnO}_{2}$ con altos contenidos de litio (17) se ha podido observar como las partículas de agregados de estaño, que se forman durante el proceso de inserción de litio en el material anódico y que poseen tamaño promedio de unos $100 \mathrm{~nm}$, se encuentran envueltas por una capa de unos 30-40 ̊ formada por productos procedentes de la reacción de descomposición del electrólito. Esta capa actúa como una interfase de electrólito sólido $(18,19)$, en la cual existe conducción de iones litio y actúa como aislante electrónico, lo que hace perder el contacto electrónico entre los agregados de estaño.

$\mathrm{Si}$ bien los materiales compuestos amorfos de Sn-B-P-O han sido los primeros materiales de estaño estudiados para su uso como ánodos en baterías ion-litio (10) y, a su vez, han mostrado unos buenos resultados electroquímicos, son también variadas las soluciones propuestas para la optimización de las propiedades electroquímicas de esta familia de compuestos. Tres métodos de actuación merecen ser comentados i) diferente forma de preparación del óxido de estaño; ii) dopaje con otros elementos; iii) formación de nuevas aleaciones.

Diferentes métodos de síntesis se han utilizado para la obtención de $\mathrm{SnO}_{2}$, con la finalidad de obtener un material con un tamaño de partícula lo más pequeño posible, favorable para inhibir la formación de grandes agregados de estaño. De este modo, se ha ensayado con éxito la formación de películas 
delgadas $(20-22)$ y muestras nanocristalinas $(12,23)$ de óxido de estaño, demostrándose que no es necesario disponer de un material amorfo, ni de un óxido mixto, para poder obtener un buen rendimiento electroquímico. Brousse y col. (20) prepararon películas delgadas de $\mathrm{SnO}_{2}$ empleando el método de deposición química en fase de vapor a baja presión. De este modo consiguieron preparar muestras con tamaños cristalinos de unos 30-50 nm. Las películas delgadas de $\mathrm{SnO}_{2}$ mostraron un excelente comportamiento electroquímico como electrodos, desarrollando una capacidad de unos $500 \mathrm{Ah} / \mathrm{kg}$ durante unos cien ciclos. Nam y col. (21) obtuvieron resultados electroquímicos similares con ánodos de $\mathrm{SnO}_{2}$ depositados en forma de películas delgadas mediante evaporación por haz de electrones. Este grupo realizó un estudio detallado de la influencia de la temperatura de calcinación en la morfología y estructuras de las películas preparadas. Las mejores propiedades electroquímicas se encontraron en ánodos calcinados a temperaturas de unos $600{ }^{\circ} \mathrm{C}$. En estas condiciones, las partículas son cristalinas y las láminas poseen una buena adherencia al sustrato, con espesores inferiores a $0.5 \mu \mathrm{m}$. Películas más gruesas $(>1 \mu \mathrm{m})$ mostraron una pérdida continua de capacidad reversible asociada a la existencia de una alta resistencia interna.

El dopaje de óxidos de estaño con diferentes elementos es otra de las alternativas que puede ser ensayada con el objetivo de mejorar las propiedades electroquímicas de estos materiales. La investigación de nuestro grupo de trabajo se ha centrado en esta línea de actuación y los primeros estudios se han realizado en los sistemas $\mathrm{SnO}_{2} / \mathrm{M}(\mathrm{M}=\mathrm{Mo}$, In, B). La elección del molibdeno se debe al favorable efecto que ejerce este elemento en las propiedades electrocrómicas del $\mathrm{SnO}_{2}$ (24). Asimismo, el óxido de indio dopado con estaño (ITO) también es un buen material electrocrómico (25). En cuanto al boro,
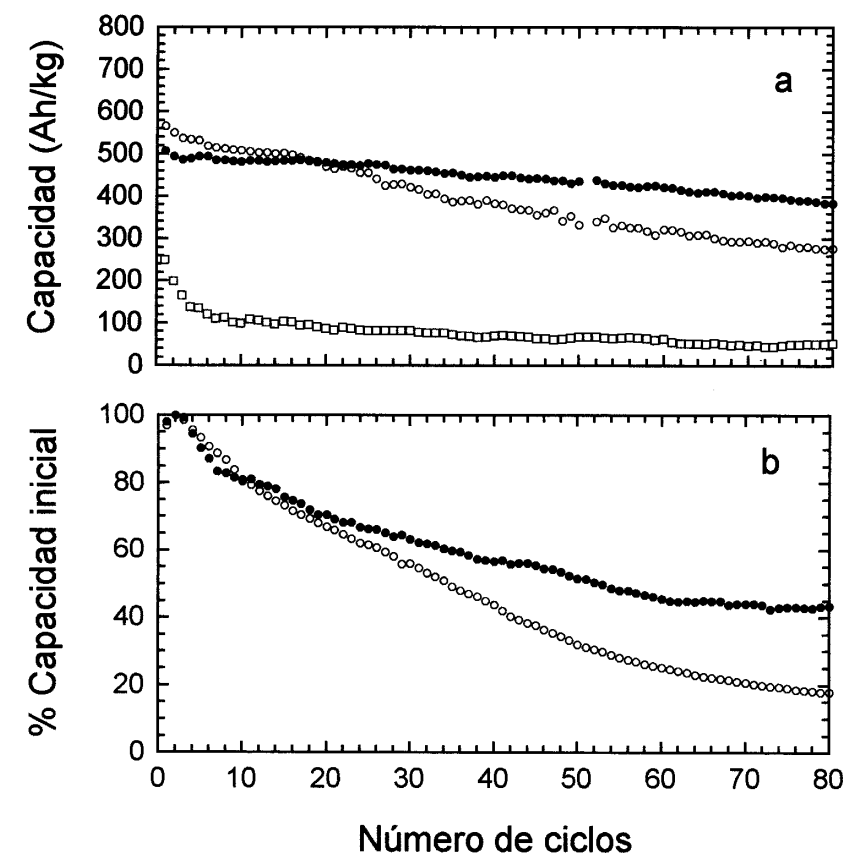

Figura 3: Evolución de la capacidad específica desarrollada por los electrodos de estaño frente al número de ciclos en (a) baterías secundarias de litio: $\mathrm{SnO}_{2} / \mathrm{LiPF}_{6}$ (EC-DMC)/ $\mathrm{Li}(\mathrm{o}),\left[\mathrm{SnO}_{2} / \mathrm{Mo}(2 \%)\right] / \mathrm{LiPF}_{6}$ $\left(\right.$ EC-DMC) $/ \mathrm{Li}(\bullet)$ y $\left[\mathrm{SnO}_{2} / \mathrm{Mo}(30 \%)\right] / \mathrm{LiPF}_{6}(\mathrm{EC}-\mathrm{DMC}) / \mathrm{Li}(\square) ; \mathrm{y}(\mathrm{b})$ en baterías ion-litio: $\mathrm{Li}_{0.73} \mathrm{Mn}_{1.97} \mathrm{O}_{4} / \mathrm{LiPF}_{6}(\mathrm{EC}-\mathrm{DMC}) / \mathrm{SnO}_{2}$ (o), $\left.\mathrm{Li}_{0.73} \mathrm{Mn}_{1.97} \mathrm{O}_{4}\right] / \mathrm{LiPF}_{6}(\mathrm{EC}-\mathrm{DMC}) /\left[\mathrm{SnO}_{2} / \mathrm{Mo}(2 \%)\right](\bullet)$. como se ha señalado en párrafos anteriores, ejerce un efecto favorable en óxidos que contienen enlaces Sn(II)-O como sitios activos para la inserción de litio. Como métodos de síntesis se han utilizado condiciones hidrotermales a temperaturas moderadas $\left(150{ }^{\circ} \mathrm{C}\right),(23,26)$, apropiadas para obtener óxidos de baja cristalinidad, con un tamaño de partícula nanométrico, factor que facilita la reversibilidad de la reacción con el litio. Los ánodos de $\mathrm{SnO}_{2}$ dopados con molibdeno (23) muestran una mejora ostensible en el mantenimiento de la capacidad generada (Figura 3). Esta mejora, que se manifiesta sólo para bajos porcentajes de molibdeno, es originada, probablemente, por la influencia que ejerce el molibdeno sobre los hábitos de crecimiento cristalino del óxido, así como por la dispersión que origina entre los átomos de estaño. El molibdeno presente, probablemente en forma de $\mathrm{MoO}_{3^{\prime}}$, permanece inactivo frente al proceso de inserción de litio. En cambio, en los óxidos mixtos de $\mathrm{SnO}_{2} / \mathrm{B}$ y SnO 2 / In (26) se observa una pérdida continua de la capacidad reversible suministrada por el electrodo conforme se realizan sucesivos ciclos de carga/descarga. Aun cuando no disponemos de una explicación clara del origen de esta diferencia de comportamiento, sugerimos que el efecto negativo de estos agentes dopantes se podría asociar al escaso rendimiento electroquímico de los propios óxidos $\mathrm{B}_{2} \mathrm{O}_{3}$ y $\mathrm{In}_{2} \mathrm{O}_{3}$ , los cuales son activos a la inserción electroquímica de litio, más que a factores tales como tamaño, forma o cristalinidad de las partículas.

Por otra parte, óxidos $\mathrm{SnO}_{\mathrm{x}}$ dopados con silicio (27) mostraron una disminución en la capacidad irreversible, en relación con el $\mathrm{SnO}_{2}$ debido a una menor presencia de oxígeno en el material electródico por efecto del dopaje. La presencia de silicio parece favorecer la formación y estabilización de aleaciones de muy alto contenido de litio, $\mathrm{Li}_{6} \mathrm{Sn}$, que aumentan la cantidad de $\mathrm{Li}^{+}$que puede ser insertado/desinsertado reversiblemente.

\subsubsection{ALEACIONES DE ESTAÑO.}

El éxito del buen comportamiento de los óxidos compuestos amorfos de estaño, patentados por investigadores de Fuji Photo Film Co. (3), radica en que estos materiales pueden ser convertidos, en condiciones cercanas al equilibrio, en alea-

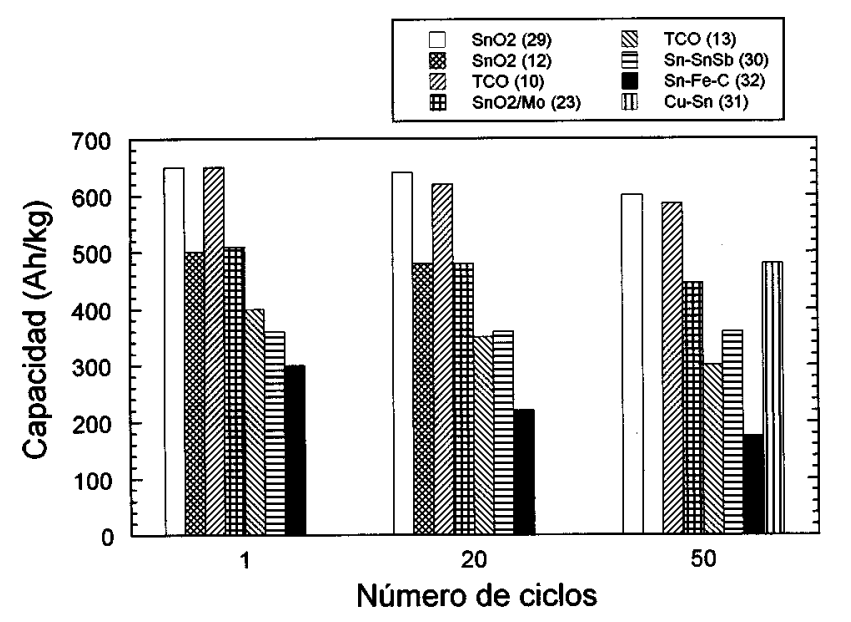

Figura 4: Evolución de la capacidad específica desarrollada por diversos ánodos de estaño en función del proceso de ciclación electroquímica. Entre paréntesis se indica la referencia correspondiente. 
ciones de Li-Sn durante el proceso electroquímico de descarga. Por tanto, sus propiedades son esencialmente las correspondientes a las que presentan las aleaciones binarias de litio. Esto ha originado que numerosos grupos de investigación hayan reexaminado posibles metales susceptibles de almacenar de modo reversible grandes cantidades de litio, mediante procesos de aleación, para usarlos como sustitutos de los materiales carbonáceos en los electrodos negativos de las baterías ion-litio $(7,28,29)$. Los estudios se han centrado principalmente en aleaciones de estaño, las cuales no presentan la capacidad irreversible que aparecía en los óxidos como consecuencia de la formación de $\mathrm{Li}_{2} \mathrm{O}$. Sin embargo, la inserción/desinserción de litio en un metal, como por ejemplo estaño (22), origina grandes cambios de volumen que producen la fragmentación y desmoronamiento del material activo, con la consiguiente pérdida de contacto electródico entre las partículas individuales. La optimización de estos materiales electródicos se ha llevado a cabo a través de dos tipos de actuaciones principalmente. i) Se ha realizado un control textural y morfológico de los materiales sintetizados, encontrándose que aquellos ánodos que poseen pequeños tamaños de partículas $(200-400 \mathrm{~nm})$ reducen considerablemente la fragmentación del electrodo y mejoran sensiblemente sus propiedades electroquímicas (28). ii) Se han sintetizado materiales electródicos multifase de pequeño tamaño de partícula. La mejora de la reversibilidad en el mecanismo de inserción de litio en ánodos multifásicos puede originarse de dos modos, que dependen de los componentes del electrodo. Así puede ocurrir: i) que ambos componentes reaccionen electroquímicamente para insertar/desinsertar litio, lo cual acontece a potenciales diferentes de tal modo que cuando un componente se expande por formación de su aleación con litio, el otro actúa de amortiguador evitando la fragmentación de las aleaciones que se forman, y viceversa (vgr. Sn-SnSb (30)); o bien ii) que uno de los componentes forme aleaciones con el litio, el caso del estaño, y los otros sean inactivos frente a la reacción electroquímica, actuando como material dispersante de las aleaciones $\mathrm{Li} / \mathrm{Sn}$ (vgr. $\mathrm{Cu}_{6} \mathrm{Sn}_{5}(31), \mathrm{Sn}_{2} \mathrm{Fe}_{-} \mathrm{SnFe}_{3} \mathrm{C}$ (32-35)).

Finalmente, en la Figura 4 se comparan las capacidades desarrolladas por diversos electrodos de estaño, tanto óxidos como aleaciones, correspondientes a la reacción de inserción de litio durante el primer ciclo y los ciclos número veinte y cincuenta. De esta manera se puede fácilmente visualizar y resumir la retención de la capacidad que presenta esta variada familia de materiales anódicos.

\section{CONCLUSIONES}

Los óxidos de estaño son capaces de actuar como materiales anódicos en baterías de ion-litio y ser competitivos frente a los materiales carbonáceos utilizados en las baterías comerciales. La reacción de inserción de litio en el $\mathrm{SnO}_{2}$ conduce a la formación de $\mathrm{Li}_{2} \mathrm{O}$ y aleaciones $\mathrm{Li}-\mathrm{Sn}$, las cuales poseen la propiedad de almacenar litio en forma reversible. Para la optimización del rendimiento electroquímico de estos materiales se requiere un control y prevención de la formación de agregados de átomos de estaño, que se puede realizar de formas diversas: i) mediante la introducción de elementos en la red de los óxidos, que aun siendo inactivos a la inserción de litio, ayuden a la dispersión de los átomos de estaño; ii) disminución del tamaño de partícula y iii) control del rango de potencial en el cual se lleva a cabo el proceso de inserción/desinserción de litio. Por otro lado, aleaciones metálicas de estaño con diversos elementos $(\mathrm{Sb}, \mathrm{Cu}, \mathrm{Fe})$ también muestran comportamientos electroquímicos prometedores en su actuación como ánodos en baterías de ion-litio.

\section{AGRADECIMIENTOS.}

Este trabajo ha sido realizado gracias a las ayudas económicas concedidas por el Ministerio de Educación y Cultura (CICyT Proyecto PB95-0561) y la Junta de Andalucía (Grupo FQM-175).

\section{BIBLIOGRAFÍA}

1 R. Owen, «Rechargeable lithium batteries», Chem. Soc. Rev., 26, 259-267 (1997).

2 M.Winter, J.O. Besenhard, M.E. Spahr, P. Novák, «Insertion electrode materials for rechargeable lithium batteries», Adv. Mater., 10 (10), 725-763 (1998).

3 Y. Idota, M. Nishima, Y. Miyaki, T. Kubota, T. Misayaki, «Nonaqueous secondary battery", Eur. Patent. 0651450A1 (1994).

4 B. Orel, U. Lavrencic-Stangar, K. Kalcher, «Electrochemical and structural properties of $\mathrm{SnO}_{2}$ and $\mathrm{Sb}: \mathrm{SnO}_{2}$ transparent electrodes with mixed electronically conductive and ion storage characteristics ", J. Electrochem. Soc., 141, L127 (1994).

5 P.Olivi, E.C. Pereira, E. Longo, J.A. Varelly, y L.O. de S.Bulhoes, «Preparation and characterization of dip-coated $\mathrm{SnO}_{2}$ film for transparent electrodes for transmissive electrochromic devices», J. Electrochem. Soc., 140. L81 (1993).

6 C. J. Wen, R.A. Huggins, «Thermodinamic study of the lithium-tin system», J. Electrochem. Soc., 128 (6), 1181-1187, (1981).

7 R.A. Huggins, «Alloy negative electrodes for lithium batteries formed in-situ from oxides», Ionics, 3, 245-255 (1997).

8 I.A. Courtney, J.R. Dahn, «Electrochemical and in situ X-ray diffraction studies of the reaction of lithium with tin oxide composites», J. Electrochem. Soc., 144 (6), 2045-2052 (1997).

9 W. Liu, X. Huang, Z. Wang, H. Li, L. Chen, «Studies of stannic oxide as an anode material for lithium-ion batteries», J. Electrochem. Soc., 145 (1), 59-62 (1998).

10 Y. Idota, T. Kubota, A. Matsufuji, Y. Maekawa, T. Miyasaka, «Tin-based amorphous oxide: a high-capacity lithium-ion-storage material», Science, 276, 1395-1397 (1997).

11 Y. Wang, J. Sakamoto, C.K. Huang, S. Surampudi, S.G. Greenbaum, «Lithium7 NMR investigation of electrochemical reaction of lithium with $\mathrm{SnO»,} \mathrm{Solid}$ State Ionics, 110, 167-172 (1998).

12 I.A. Courtney, J.R. Dahn, «Key factors controlling the reversibility of the reaction of lithium with $\mathrm{SnO}_{2}$ and $\mathrm{Sn}_{2} \mathrm{BPO}_{6}$ glass», J. Electrochem. Soc., 144 (9), 2943-2948 (1997)

13 I.A. Courtney, W.R. McKinnon, J.R. Dahn, «On the aggregation of tin in SnO composite glasses caused by the reversible reaction with lithium», J. Electrochem. Soc., 146 (1), 59-68 (1999).

14 W.G. Moffat, «The handbook of binary phase diagrams», Schenectady, Genium Publishing Co., N.Y. (1990).

15 S. Machill, T. Shodai, Y. Sakurai, J. Yamaki «Electrochemical and structural investigations of the reaction of lithium win tin-based composite oxide glasses», J. Solid State Electrochem., 3, 97-103 (1999).

$16 \mathrm{H}$. Li, X. Huang, L. Chen, «Direct imaging of the passivating film and microstructure of nanometer-scale $\mathrm{SnO}$ anodes in lithium rechargeable batteries», Electrochem. Solid-State Lett., 1 (6), 241-243 (1998)

17 R. Retoux, T. Brousse, D.M. Schleich, «High resolution electron microscopy investigation of capacity fade in $\mathrm{SnO}_{2}$ electrodes for lithium ion batteries», J. Electrochem. Soc., 146 (7) 2472 (1999).

$18 \mathrm{H}$. Li, J. Li, X. Huang, L. Chen, «Formation process of passivation layer on $\mathrm{SnO}$ anode», 9th International Meeting on Lithium Batteries, P.G. Bruce, The Electrochemical Society, Julio 1998

$19 \mathrm{H}$. Li, X. Huang, L. Chen, «EIS study of SnO and nano-SnO anodes in lithium rechargeable batteries», 9th International Meeting on Lithium Batteries, P.G. Bruce, The Electrochemical Society, Julio 1998.

20 T. Brousse, R. Retoux, U. Herterich, D.M. Schleich, «Thin-Film crystalline $\mathrm{SnO}_{2}$-lithium electrodes», J. Electrochem. Soc., 145 (1), 1-4 (1998).

21 S.C. Nam, Y.H. Kim, W.I. Cho, B.W. Cho, H.S. Chum, K.S. Yun, «Charge-discharge performance of electron-beam-deposited tin oxide thin-film electrodes», Electrochem. Solid-State Lett., 2 (1), 9-11 (1999).

22 R. Le Gall, V. Hays, T. Brousse, D.M. Schleich, «Metallic tin anodes for lithium ion batteries», 9th International Meeting on Lithium Batteries, P.G. Bruce, The Electrochemical Society, Julio 1998. 
23 J. Morales, L. Sánchez, «Improving the electrochemical performance of $\mathrm{SnO}_{2}$ cathodes in lithium secondary batteries by doping with Mo», J. Electrochem. Soc., 146 (5), 1640-1642 (1999).

24 U.O. Krasovec, B. Orel, S. Hocevar, Y. Musevic, «Electrochemical and spectroelectrochemical properties of $\mathrm{SnO}_{2}$ and $\mathrm{SnO}_{2} / \mathrm{Mo}$ transparent electrodes with high ion-storage capacity», J. Electrochem. Soc., 144(10), 3398-3409 (1997).

25 P.M.M.C. Bressers, E.A. Meulenkamp, «The electrochemical behavior of indium tin oxide in propilene carbonate solutions», J. Electrochem. Soc., 145(7), 2225-2230 (1998)

26. J. Morales, L. Sánchez, «Electrochemical behaviour of $\mathrm{SnO}_{2}$ doped with boron and indium in anodes for lithium secondary batteries», Solid State Ionics.

27. H. Huang, E.M. Kelder, J. Schoonman, L. Chen, «Electrochemical characteristics of $\mathrm{Si}$ doped $\mathrm{SnO}_{\mathrm{x}}$ as anode for lithium ion batteries», 9th International Meeting on Lithium Batteries, P.G. Bruce, The Electrochemical Society, Julio 1998

28. J. O. Besenhard, J. Yang, M. Winter, «Will advanced Li-alloy anodes have a chance in lithium-ion batteries?» 8th International Meeting on Lithium Batteries, O. Yamamoto, The Electrochemical Society of Japan, Junio 1996.
29. T. Brousse, D. Defives, L. Pasquereau, S.M. Lee, U. Herterich, D.M. Schleich, «Metal oxide anodes for Li-ion batteries», Ionics, 3, 332-337 (1997)

30. J. Yang, M. Wachtler, M. Winter, J.O. Besenhard «Sub-microcrystalline Sn and Sn-SnSb powders as lithium storage materials for lithium-ion batteries», Electrochem. Solid-State Lett., 2 (4), 161-163 (1999).

31. J.T. Vaughey, K.D. Kepler, D.R. Vissers, M.M. Thackeray «Copper-tin anodes for rechargeable lithium batteries», 9th International Meeting on Lithium Batteries, P.G. Bruce, The Electrochemical Society, Julio 1998.

32. O. Mao, R.L. Turner, I.A. Courtney, B.D. Fredericksen, M.I. Buckett, L.J. Krause, J.R. Dahn, «Active/inactive nanocomposites as anodes for Li-ion batteries» Electrochem. Solid-State Lett., 2 (1), 3-5 (1999).

33. O. Mao, R.A. Dunlap, J.R. Dahn, «Mechanically alloyed Sn-Fe(-C) powders as anode materials for $\mathrm{Li}$-ion batteries. I. The $\mathrm{Sn}_{2} \mathrm{Fe}-\mathrm{C}$ system». J. Electrochem. Soc., 146 (2), 405-413 (1999).

34. O. Mao, J.R. Dahn, «Mechanically alloyed Sn-Fe(-C) powders as anode materials for Li-ion batteries. II. The Sn-Fe system». J. Electrochem. Soc., 146 (2), 414-422 (1999).

35. O. Mao, J.R. Dahn, «Mechanically alloyed Sn-Fe(-C) powders as anode materials for Li-ion batteries. III. $\mathrm{Sn}_{2} \mathrm{Fe}: \mathrm{SnFe}_{3} \mathrm{C}$ active/inactive composites». J. Electrochem. Soc., 146 (2), 423-427 (1999). 